\title{
Infinitely Many Sign-Changing Solutions for Some Nonlinear Fourth-Order Beam Equations
}

\author{
Ying $\mathrm{Wu}^{1}$ and Guodong $\mathrm{Han}^{2}$ \\ ${ }^{1}$ College of Science, Xian University of Science and Technology, Xi'an, Shaanxi 710054, China \\ ${ }^{2}$ College of Mathematics and Information Science, Shaanxi Normal University, Xian, Shaanxi 710062, China \\ Correspondence should be addressed to Guodong Han; gdhan.math@gmail.com
}

Received 5 September 2012; Accepted 22 April 2013

Academic Editor: Svatoslav Staněk

Copyright (c) 2013 Y. Wu and G. Han. This is an open access article distributed under the Creative Commons Attribution License, which permits unrestricted use, distribution, and reproduction in any medium, provided the original work is properly cited.

\begin{abstract}
Several new existence theorems on positive, negative, and sign-changing solutions for the following fourth-order beam equation are obtained: $u^{(4)}=f(t, u(t)), t \in[0,1] ; u(0)=u(1)=u^{\prime \prime}(0)=u^{\prime \prime}(1)=0$, where $f \in C\left([0,1] \times \mathbb{R}^{1}, \mathbb{R}^{1}\right)$. In particular, an infinitely many sign changing solution theorem is established. The method of the invariant set of decreasing flow is employed to discuss this problem.
\end{abstract}

\section{Introduction and Main Results}

It is well known that the following fourth-order two-point boundary value problem (BVP):

$$
\begin{gathered}
u^{(4)}=f(t, u(t)), \quad t \in[0,1], \\
u(0)=u(1)=u^{\prime \prime}(0)=u^{\prime \prime}(1)=0
\end{gathered}
$$

describes the deformation of an elastic beam both of whose ends are simply supported at 0 and 1 . Owing to the importance of high-order differential equations both in theory and practice, much attention has been paid to such problems by a number of authors, see [1-15] and references therein.

Among these literatures, some of the authors used to deal with the existence of positive solutions by employing the cone expansion and compression fixed point theorem of norm type $[1,2,5,6]$, the five functionals fixed point theorem [10], and the abstract fixed point index theory $[3,4]$. The main assumptions imposed on the nonlinear term $f$ included that it is superlinear or sublinear in $u$, and its growth on some intervals is restricted by suitable functions, or it is asymptotically linear in 0 and $\infty$.

Various methods have been applied to these problems in recent years. In [7], by using the strongly monotone operator principle and the critical point theory, Li et al. established some sufficient conditions for $f$ to guarantee that the problem has a unique solution, at least one nonzero solution, or infinitely many solutions. In [8], some new existence theorems on multiple positive, negative, and sign-changing solutions of BVP (1) were established by combining the critical point theory and the method of sub- and supsolution. In [11], the authors obtained a three-solution theorem and an infinitely-many-solution theorem by applying the Morse theory, in which they removed a condition in [7]. In [13, 15], Yang and Zhang got some infinitely many mountain pass solutions theorems for the problems with parameters by the mountain pass theorem in order interval, in which they imposed conditions on $f$ to guarantee that the equation has infinitely many pairs sub- and supsolutions.

Besides, a few papers are concerned with the existence and multiplicity of the sign-changing solutions for kinds of fourth-order boundary value problems $[8,9,12]$ recently. One reason is from the following fact. Consider the linear eigenvalue problem

$$
\begin{gathered}
u^{(4)}=\lambda u, \quad t \in[0,1], \\
u(0)=u(1)=u^{\prime \prime}(0)=u^{\prime \prime}(1)=0 .
\end{gathered}
$$


It is known that (2) has an unbounded sequence of eigenvalues

$$
\pi^{4}<(2 \pi)^{4}<\cdots<(m \pi)^{4}<\cdots
$$

with corresponding eigenfunctions

$$
\sin \pi t, \sin 2 \pi t, \ldots, \sin m \pi t, \ldots
$$

Obviously, the first eigenfunction $\sin \pi t>0$ for all $t \epsilon$ $(0,1)$ and other eigenfunctions $\sin m \pi t(m=2,3, \ldots)$ are all sign-changing functions on $[0,1]$. This fact suggests that BVP (1) regarded as a nonlinear perturbation of (2) should have more sign-changing solutions than positive and negative solutions. Another reason is that sign-changing solutions should have more complicated properties, such as the times of changing sign. Thus, sign-changing solutions are interesting challenges in mathematics. In a word, the study on signchanging solutions is the natural extension and deepening of the previous research on positive solutions. In [12], using the fixed point index and the critical group, Li et al. obtained that the fourth-order Neumann problem has at least one positive solution and two sign-changing solutions under certain conditions. In [9], Li proved that the fourth-order problem in which $f$ contains the bending moment term $u^{\prime \prime}$ has multiple sign-changing solutions by the fixed point index theory in cones and an antisymmetrical extension method of solution.

In the present paper, motivated by the above results, we investigate the positive, negative, and sign-changing solutions for BVP (1) as well. By applying the method of the invariant set of decreasing flow, we establish several multiple solutions theorems. Furthermore, an infinitely many sign changingsolution theorem is obtained. The comparisons with the results in the literatures are stated in the remarks below our main theorems. And we present four simple examples to which our theorems can be applied, respectively.

For convenience, we list our conditions as follows:

$\left(\mathrm{H}_{1}\right) f \in C\left([0,1] \times \mathbb{R}^{1}, \mathbb{R}^{1}\right)$,

$\left(\mathrm{H}_{2}\right) \limsup _{u \rightarrow 0}|f(t, u) / u|<\pi^{4}$ for all $t \in[0,1]$ uniformly,

$\left(\mathrm{H}_{3}\right)$ there exist $\mu \in(0,1 / 2)$ and $M>0$ such that

$F(t, u) \triangleq \int_{0}^{u} f(t, v) \mathrm{d} v \leqslant \mu u f(t, u) \quad \forall|u| \geqslant M, t \in[0,1]$

$\left(\mathrm{H}_{4}\right) f(t, 0)=0$ for all $t \in[0,1]$,

$\left(\mathrm{H}_{5}\right)$ there exist two real numbers $c>0$ and $q>2$ such that

$$
|f(t, u)| \leqslant c\left(1+|u|^{q-1}\right) \quad \forall(t, u) \in[0,1] \times \mathbb{R}^{1},
$$

$\left(\mathrm{H}_{6}\right) f(t, u)$ is odd in $u$, that is, $f(t,-u)=-f(t, u)$ for all $(t, u) \in[0,1] \times \mathbb{R}^{1}$.
The following three conditions are a little weaker than $\left(\mathrm{H}_{2}\right)$ and $\left(\mathrm{H}_{3}\right)$, respectively:

$\left(\mathrm{H}_{2}^{\prime}\right) \lim \sup _{u \rightarrow 0+}(f(t, u) / u)<\pi^{4}$ for all $t \in[0,1]$ uniformly,

$\left(\mathrm{H}_{2}^{\prime \prime}\right) \limsup _{u \rightarrow 0}(f(t, u) / u)<\pi^{4}$ for all $t \in[0,1]$ uniformly,

$\left(\mathrm{H}_{3}^{\prime}\right)$ there exist $\mu \in(0,1 / 2)$ and $M>0$ such that

$$
F(t, u) \triangleq \int_{0}^{u} f(t, v) d v \leqslant \mu u f(t, u) \quad \forall u \geqslant M, t \in[0,1] .
$$

Now, the main results can be stated as follows.

Theorem 1. Assume that $\left(H_{1}\right),\left(H_{2}^{\prime}\right)$, and $\left(H_{3}^{\prime}\right)$ hold. In addition, $f(t, u) \geqslant 0$ for all $u \in[0,+\infty)$. Then, $B V P$ (1) has at least a positive solution in $C^{4}[0,1]$.

Remark 2. We will apply the method of the invariant set of decreasing flow to prove this theorem in Section 3. When dealing with BVP (1) by the cone expansion and compression theorems $[16,17]$, we usually assume that

$$
\limsup _{u \rightarrow 0+} \frac{f(t, u)}{u}=0, \quad \limsup _{u \rightarrow+\infty} \frac{f(t, u)}{u}=+\infty .
$$

The first equation is stronger than $\left(\mathrm{H}_{2}^{\prime}\right)$, while the latter is weaker than $\left(\mathrm{H}_{3}\right)$. So, both of the two methods have their own characteristics.

Example 3. Let

$$
\begin{aligned}
f & (t, u) \\
& = \begin{cases}a u+b(t) \ln (1+u) \arctan u+c|u|^{\gamma} u, & (t, u) \in[0,1] \times[0,+\infty), \\
0, & (t, u) \in[0,1] \times(-\infty, 0),\end{cases}
\end{aligned}
$$

where $a \in\left[0, \pi^{4}\right), b \in C([0,1],[0,+\infty))$ and $c, \gamma>0$. It is easy to check that all conditions of Theorem 1 are satisfied. So, BVP (1) with the nonlinear term (9) has at least a positive solution.

Theorem 4. Assume that $\left(H_{1}\right),\left(H_{2}^{\prime \prime}\right)$, and $\left(H_{3}\right)$ hold. In addition, $f(t, u) u \geqslant 0$ for all $u \in \mathbb{R}^{1}$. Then, $B V P$ (1) has at least a positive solution and a negative solution in $C^{4}[0,1]$.

Remark 5. In [7], using the mountain pass lemma, Li et al. obtained that BVP (1) has at least one nonzero solution in $C^{4}[0,1]$ under the assumptions that $\left(\mathrm{H}_{1}\right),\left(\mathrm{H}_{2}^{\prime \prime}\right)$, and $\left(\mathrm{H}_{3}\right)$ hold [7, Theorem 3.3]. By adding a condition $f(t, u) u \geqslant 0$, we get two solutions, one positive and the other negative. So, Theorem 4 can be seen as a complement of [7, Theorem 3.3].

Example 6. Let

$$
\begin{array}{r}
f(t, u)=a \arctan u+b(t) u\left|e^{u}-1\right|+c|u|^{\gamma} u \\
\qquad \text { for }(t, u) \in[0,1] \times \mathbb{R}^{1},
\end{array}
$$


where $a \in\left[0, \pi^{4}\right), b \in C([0,1],[0,+\infty))$ and $c, \gamma>0$. It is easy to verify that all conditions of Theorem 4 are satisfied. So, BVP (1) with the nonlinear term (10) has at least two solutions, one positive and the other negative. Reference [7, Theorem 3.3] can only guarantee a nonzero solution for this example.

Theorem 7. Assume that $\left(H_{1}\right)-\left(H_{5}\right)$ hold. Then, BVP (1) has at least a positive solution, a negative solution, and a signchanging solution in $C^{4}[0,1]$.

Remark 8. Theorem 7 can be regarded as an improvement of $\left[8\right.$, Corollary 18] though we add a growth condition $\left(\mathrm{H}_{5}\right)$. Firstly, the nonlinear term $f$ here only needs to be continuous while $f$ is locally Lipschitz continuous with respect to $u$ and strictly increasing in [8]. Secondly, as is known, when the method of the invariant set of decreasing flow is applied to differential equations, the main difficulty is that the cone has an empty interior in the function space we work in, such as the positive cone in $L^{2}[0,1]$. Generally, one needs the $E$-regular operator or the bootstrap argument [8]. In our proof of this theorem, from the idea of $[18,19]$, we construct open sets in $L^{2}[0,1]$ directly instead of introducing $C_{0}[0,1] \hookrightarrow L^{2}[0,1]$ as in $[8]$.

Example 9. Let

$$
\begin{array}{r}
f(t, u)=a u+b(t)|\sin u| \arctan u+c u^{4} \\
\text { for }(t, u) \in[0,1] \times \mathbb{R}^{1}
\end{array}
$$

where $a \in\left[0, \pi^{4}\right), b \in C[0,1]$ and $c>0$. It is easy to verify that all conditions of Theorem 7 are satisfied. So, Theorem 7 ensures that BVP (1) with the nonlinear term (11) has at least a positive solution, a negative solution, and a signchanging solution. Since neither $f(t, u)$ nor $f(t, u)+m u$ is strictly increasing, Corollary 18 in [8] cannot be applied to this example.

Theorem 10. Assume that $\left(H_{1}\right)-\left(H_{3}\right),\left(H_{5}\right)$, and $\left(H_{6}\right)$ hold. Then, BVP (1) has infinitely many sign-changing solutions in $C^{4}[0,1]$.

Remark 11. Using a symmetric mountain pass lemma [20, Theorem 9.12] due to Rabinowitz, Li et al. obtained an infinitely many solutions for BVP (1) [7, Theorem 3.4]. In [11], we obtained a similar conclusion [11, Theorem 1.3] after removing condition of [7, Theorem 3.4] and strengthening the differentiability of $f$. Yang and Zhang $[13,15]$ established some infinitely many mountain pass solutions theorems for the fourth-order boundary value problems with parameters by the mountain pass theorem in order interval, in which they supposed that $f$ is strictly increasing in $u$, and the problem has infinitely many pairs of sub- and supsolutions, such as the following $\left[15\right.$, condition $\left.\left(\mathrm{H}_{3}\right)\right]$.

There exist sequences $\left\{\alpha_{i}\right\},\left\{\beta_{i}\right\} \subset C_{0}[0,1]$ satisfying

$$
\begin{aligned}
0 & <\alpha_{1}<\beta_{1}<\cdots<\alpha_{i}<\beta_{i}<\alpha_{i+1}<\beta_{i+1} \\
& <\cdots<\alpha_{n}<\beta_{n}<\cdots
\end{aligned}
$$

and $\left\{\alpha_{i}, \beta_{i}\right\}(i=1,2, \ldots)$ is a pair of strict subsolution and supsolution of BVP....

This condition seems somewhat strong. Actually, it is not easy to impose conditions on the nonlinear term $f$ to guarantee that $\left[15\right.$, condition $\left.\left(\mathrm{H}_{3}\right)\right]$ holds. Besides, in $[7,11$, $13,15]$, though the authors have obtained the existence of infinitely many solutions, they have not given the signs of them. In fact, to our knowledge, none of the infinitely-manysign-changing-solution theorem for BVP (1) has been found in the literatures so far. In contrast to [7, Theorem 3.4] and [11, Theorem 1.3], by adding a growth condition $\left(\mathrm{H}_{5}\right)$, Theorem 10 gets more information for those infinite solutions; that is, they all change their signs in the interval $[0,1]$. Compared with the theorems in $[13,15]$, our conditions are more natural and easier to verify.

Example 12. Let

$$
\begin{array}{r}
f(t, u)=a \tan u+b(t) \arctan u \ln \left(1+u^{2}\right)+c|u|^{\gamma} u \\
\qquad \text { for }(t, u) \in[0,1] \times \mathbb{R}^{1},
\end{array}
$$

where $a \in\left[0, \pi^{4}\right], b(t) \in C[0,1]$ and $c, \gamma>0$. It is easy to verify that all conditions of Theorem 10 are satisfied. So, Theorem 10 ensures that BVP (1) with the nonlinear term (13) has infinitely many sign-changing solutions. Theorem 3.4 in [7] and Theorem 1.3 in [11] can also guarantee that the problem has infinitely many solutions but cannot get their signs.

This paper is organized as follows. In Section 2, we recall some facts on the method of the invariant set of descending flow and prove two useful abstract theorems. The main results are proved in Section 3.

\section{Preliminaries}

In this section, we firstly outline some basic concepts on the method of the invariant set of descending flow. Secondly, four theorems which will be used in the proofs of our main results are listed. Among them, two are our new results, and the other two are due to [19]. Please refer to [21, 22] for more details about the method of the invariant set of descending flow.

Let $X$ be a real Banach space, $J$ a $C^{1}$ functional defined on $X, J^{\prime}(u)$ the gradient operator of $J$ at $u \in X$, and $W$ a pseudogradient vector field for $J$. Let

$$
\operatorname{Cr}(J)=\left\{u \in X: J^{\prime}(u)=\theta\right\}, \quad X_{0}=X \backslash \operatorname{Cr}(J) .
$$

For $u_{0} \in X_{0}$, consider the following initial problem in $X_{0}$ :

$$
\begin{gathered}
\frac{\mathrm{d}}{\mathrm{d} t} \varphi(t)=-W(\varphi(t)), \quad t \geqslant 0, \\
\varphi(0)=u_{0} .
\end{gathered}
$$

By the theory of ordinary differential equations in Banach space, (15) has a unique solution in $X_{0}$, denoted by $\varphi\left(t, u_{0}\right)$, with the right maximal interval of existence $\left[0, \eta\left(u_{0}\right)\right)$. Note that $\eta\left(u_{0}\right)$ may be either a positive number or $+\infty$. It is easy to see that $J\left(\varphi\left(t, u_{0}\right)\right)$ is monotonically decreasing on $\left[0, \eta\left(u_{0}\right)\right)$, so $\varphi\left(t, u_{0}\right)$ is called a descending flow curve. 
Definition 13 (see [21]). A nonempty subset $M$ of $E$ is called an invariant set of descending flow of $J$ if

$$
\left\{\varphi\left(t, u_{0}\right): 0 \leqslant t<\eta\left(u_{0}\right)\right\} \subset M
$$

for all $u_{0} \in M \backslash \mathrm{Cr}(J)$.

Definition 14 (see [21]). Let $M$ and $D$ be invariant sets of descending flow of $J, D \subset M$. Denote

$$
\begin{gathered}
C_{M}(D)=\left\{u_{0}: u_{0} \in D \text { or } u_{0} \in M \backslash D\right. \\
\text { and there exists } 0<t^{\prime}<\eta\left(u_{0}\right) \\
\text { such that } \left.\varphi\left(t^{\prime}, u_{0}\right) \in D\right\} .
\end{gathered}
$$

If $D=C_{M}(D)$, then $D$ is called a complete invariant set of descending flow relative to $M$.

For a subset $M$ of $X, J$ is called satisfying PS condition on $M$ if any sequence $\left\{u_{n}\right\} \subset M$ such that $\left\{J\left(u_{n}\right)\right\}$ is bounded and $J^{\prime}\left(u_{n}\right) \rightarrow 0$ as $n \rightarrow+\infty$ possesses a convergent subsequence.

Following this, we use $\partial_{A} B$, and $\operatorname{int}_{A} B$ and $\operatorname{clos}_{A} B$ denote the boundary, the interior, and the closure of the set $B$ in set $A$, respectively.

Theorem 15 (see [21]). Assume that $M$ is closed and connected and is an invariant set of descending flow for $J$, and $D$ is an open subset of $M$ and an invariant set of descending flow for $J$ as well. If $C_{M}(D) \neq M, \inf _{u \in \partial_{M} D} J(u)>-\infty$, and $J$ satisfies $P S$ condition on $M \backslash D$, then

$$
\inf _{u \in \partial_{M} C_{M}(D)} J(u) \geqslant \inf _{u \in \partial_{M} D} J(u)>-\infty
$$

$\inf _{u \in \partial_{M} C_{M}(D)} J(u)$ is a critical value of $J$, and there exists at least one point on $\partial_{M} C_{M}(D)$ corresponding to this value.

Next, we list four theorems, of which two are our new results, and two are due to [19].

Assume that $H$ is a real Hilbert space, $P$ is a positive cone in $H$, and the partial order on $H$ is given by $P$. $J$ is a $C^{1}$ functional on $H$, and $J^{\prime}(u)$ can be expressed in the form of $J^{\prime}(u)=u-A u$.

Theorem 16. Suppose that $J$ satisfies $P S$ condition on $P$ and $A: P \rightarrow P . D$ is an open convex subset of $P$ and $A\left(\partial_{P} D\right) \subset D$. If there exists $u_{0} \in P \backslash D$ such that $\inf _{u \in \partial_{P} D} J(u)>J\left(u_{0}\right)$, then $J$ has at least a positive critical point.

Proof. According to [21, Lemma 2.5], since $A: P \rightarrow P$ and $A\left(\partial_{P} D\right) \subset D$, one can construct a pseudogradient vector field $W$ for $J$ such that $P$ and $D$ both are invariant sets of descending flow for $J$ determined by $W$. We only need to show $C_{P}(D) \neq P$. In fact, $u_{0} \notin C_{P}(D)$. Otherwise, if $u_{0} \in$ $C_{P}(D)$, then there exists $0<t^{\prime} \leqslant \eta\left(u_{0}\right)$ such that $\varphi\left(t^{\prime}, u_{0}\right) \in$ $D$. Then, we can find $0<t_{1}<t^{\prime}$ with $\varphi\left(t_{1}, u_{0}\right) \in \partial_{P} D$. Thus, $J\left(u_{0}\right) \geqslant J\left(\varphi\left(t_{1}, u_{0}\right)\right)$, which contradicts the fact that $J\left(u_{0}\right)<\inf _{u \in \partial_{p} D} J(u)$. Theorem 15 implies the conclusion. The proof is completed.
Remark 17. In contrast to the cone expansion and compression theorems, the operator $A$ needs not to be completely continuous, and $D$ needs not to be bounded as well. But $A$ is a gradient operator, and $J$ satisfies PS condition. These facts indicate that both methods have their own characteristics.

By symmetry, we can easily obtain the following theorem, and we omit its proof.

Theorem 18. In addition to all the conditions of Theorem 16, suppose that $J$ satisfies $P S$ condition on $-P$, and $A:-P \rightarrow-P$, $D_{1}$ is an open convex subset of $-P$ and $A\left(\partial_{-P} D_{1}\right) \subset D_{1}$. If there exists $u_{1} \in-P \backslash D_{1}$ such that $\inf _{u \in \partial_{-} D} J(u)>J\left(u_{1}\right)$, then $J$ has at least two critical points, one is positive, and the other is negative.

The following two theorems are due to [19]. For ease of use in Section 3, here we write their special cases. See [19] for more general results.

Let $\mathscr{D}^{ \pm}$be two closed convex subsets of $H$. We need the following assumptions:

$$
\begin{aligned}
& \left(\mathrm{A}_{1}\right) \mathcal{O}=\operatorname{int}_{H^{\prime}} \mathscr{D}^{+} \cap \operatorname{int}_{H^{-}} \mathscr{D}^{-} \neq \emptyset, \\
& \left(\mathrm{A}_{2}\right) A\left(\mathscr{D}^{ \pm}\right) \subset \operatorname{int}_{H} \mathscr{D}^{ \pm} \text {, } \\
& \left(\mathrm{A}_{3}\right) \text { there exists a path } h:[0,1] \rightarrow H \text { such that } h(0) \in \\
& \left(\text { int }_{H} \mathscr{D}^{+}\right) \backslash \mathscr{D}^{-}, h(1) \in\left(\text { int }_{H} \mathscr{D}^{-}\right) \backslash \mathscr{D}^{+} \text {and } \\
& \max _{s \in[0,1]} J(h(s))<\alpha_{0}=\inf _{u \in \mathscr{D}^{+} \cap \mathscr{D}^{-}} J(u),
\end{aligned}
$$

$\left(\mathrm{A}_{4}\right)$ there exist a number $\alpha_{1}$, a sequence $\left\{H_{n}\right\}$ of subspaces of $H$, and a sequence $\left\{R_{n}\right\}$ of positive numbers satisfying

$$
\operatorname{dim} H_{n} \geqslant n \quad \text { for } n \in \mathbb{N}, \quad \sup _{u \in H_{n} \backslash B_{n}} J(u) \leqslant \alpha_{1}<\alpha_{0} \text {, }
$$

where $B_{n}=\left\{u \in H_{n}:\|u\| \leqslant R_{n}\right\}$.

Remark 19. According to [21, Lemma 2.5], we deduce from $\left(\mathrm{A}_{1}\right)$ and $\left(\mathrm{A}_{2}\right)$ that $\mathcal{O}$ and $\mathscr{D}^{ \pm}$are the invariant sets of decreasing flow.

Theorem 20. Assume that $\left(A_{1}\right)-\left(A_{3}\right)$ hold, and $J$ satisfies $P S$ condition on $H$. Then, $J$ has a critical point in each of the four mutually disjoint sets: $\partial_{H} C_{H}(\mathcal{O}) \backslash\left(\mathscr{D}^{+} \cup \mathscr{D}^{-}\right), \partial_{H} C_{H}(\mathscr{O}) \cap$ int $_{H} \mathscr{D}^{+}, \partial_{H} C_{H}(\mathcal{O}) \cap \operatorname{int}_{H^{-}} \mathscr{D}^{-}$, and $\mathcal{O}$.

Theorem 21. Assume that $\left(A_{1}\right),\left(A_{2}\right)$, and $\left(A_{4}\right)$ hold, and $J$ is an even functional and satisfies $P S$ condition on $H$. Then, $J$ has a sequence of solutions $\left\{ \pm u_{n}\right\}$ in $\mathscr{M}=\partial_{H} C_{H}(\mathcal{O})$ । $\left(C_{H}\left(\right.\right.$ int $\left._{H} \mathscr{D}^{+}\right) \cup C_{H}\left(\right.$ int $\left.\left._{H} \mathscr{D}^{-}\right)\right)$such that

$$
J\left(u_{n}\right) \longrightarrow+\infty \text { as } n \longrightarrow \infty \text {. }
$$

\section{Proof of the Main Results}

In this section, we will employ the abstract theorems in Section 2 to prove Theorems 1-10.

Let $E=C[0,1]$ denote the usual real Banach space with the norm $\|u\|_{C}=\max _{t \in[0,1]}|u(t)|$ for all $u \in C[0,1]$. By $H=$ 
$L^{2}[0,1]$ we denote the usual real Hilbert space with the norm $\|u\|=\left(\int_{0}^{1}|u(t)| \mathrm{d} t\right)^{1 / 2}$ for $u \in H$. Let

$$
P=\{u \in H: u(t) \geqslant 0 \text { a.e. } t \in[0,1]\},
$$

and then $P$ is a cone in $H$ and has an empty interior in $H$.

Define a functional $J: H \rightarrow \mathbb{R}^{1}$ by

$$
J(u)=\frac{1}{2}\|u\|^{2}-\int_{0}^{1} F(t, K u(t)) \mathrm{d} t, \quad u \in H,
$$

where $F(t, u)=\int_{0}^{u} f(t, v) \mathrm{d} v, K u(t)=\int_{0}^{1} G(t, s) u(s) \mathrm{d} s$ and

$$
G(t, s)= \begin{cases}s(1-t), & 0 \leqslant s \leqslant t \leqslant 1 \\ t(1-s), & 0 \leqslant t \leqslant s \leqslant 1 .\end{cases}
$$

Then, it is easy to see that $J \in C^{1}\left(H, \mathbb{R}^{1}\right)$ with derivatives given by

$$
J^{\prime}(u)=u-K \mathbf{f} K u \triangleq u-A u, \quad \forall u \in H,
$$

where $(\mathbf{f} u)(t)=f(t, u(t))$ is a Nemytskii operator.

Remark 22. The following results are known. See [7] for details.

(i) $G:[0,1] \times[0,1] \rightarrow[0,1]$ is nonnegative continuous and $\max _{(t, s) \in[0,1] \times[0,1]} G(t, s)=1 / 4$.

(ii) $\mathbf{f}: E \rightarrow E$ is bounded and continuous.

(iii) $K$ is linear completely continuous as an operator both from $E \rightarrow E$ and $H$ to $H$. Moreover, $\|K\|_{\mathscr{L}(H, H)}=$ $1 / \pi^{2}$, where $\mathscr{L}(H, H)$ denotes the Banach space of all bounded linear operators from $H$ to $H$.

(iv) $K^{2}=K \circ K: H \rightarrow H$ is linear, compact, and symmetric, and the norm $\left\|K^{2}\right\|_{\mathscr{L}(H, H)}=1 / \pi^{4}$. We omit the subscript $\mathscr{L}(H, H)$ in the following.

(v) $A=K \mathbf{f} K: H \rightarrow H$ is a completely continuous operator.

(vi) According to [7, Lemma 3.1], BVP (1) has a nontrivial solution in $C^{4}[0,1]$ if and only if the functional $J$ has a nontrivial critical point in $H$ (i.e., $A$ has a nontrivial fixed point in $H$ ). More precisely, if $u \in C^{4}[0,1]$ is a solution of (1), then $v=K \mathbf{f} u$ is a critical point of $J$; on the other hand, if $v \in H$ is a critical point of $J$, then $K v$ is a solution of (1) in $C^{4}[0,1]$. Moreover, since $K$ is a positive operator, $K v$ has the same sign as $v$; that is, if $v \in H$ is a positive/negative/sign-changing critical point of $J$, then $K v$ is a positive/negative/signchanging solution of $(1)$ in $C^{4}[0,1]$, respectively.

Before proving main results, we first give several lemmas.

Lemma 23. Let $\nu=1 / \mu$.

(i) Assume that $\left(H_{1}\right)$ and $\left(H_{3}^{\prime}\right)$ hold. Then, $J$ satisfies PS condition on $P$, and there exist three positive numbers $C_{1}, C_{2}$, and $C_{3}$ such that

$$
\begin{array}{cc}
F(t, u) \leqslant \mu u f(t, u)+C_{1} & \forall(t, u) \in[0,1] \times[0,+\infty), \\
F(t, u) \geqslant C_{2}|u|^{\nu}-C_{3} \quad \forall(t, u) \in[0,1] \times[0,+\infty) .
\end{array}
$$

(ii) Assume that $\left(\mathrm{H}_{1}\right)$ and $\left(\mathrm{H}_{3}\right)$ hold. Then, $J$ satisfies PS condition on $H$, and there exist three positive numbers $C_{1}, C_{2}$, and $C_{3}$ such that

$$
\begin{gathered}
F(t, u) \leqslant \mu u f(t, u)+C_{1} \quad \forall(t, u) \in \times[0,1] \times \mathbb{R}^{1}, \\
F(t, u) \geqslant C_{2}|u|^{\nu}-C_{3} \quad \forall(t, u) \in[0,1] \times \mathbb{R}^{1} .
\end{gathered}
$$

Proof. Since the proof of (i) is analogous to that of (ii), we only need to prove (ii).

$J$ is the special case of $J_{1}$ defined in (53) as $m=0$ and $K_{1}=K$. See the proof of Lemma 24 for the fact that $J$ satisfies PS condition.

Since $v F(t, u)-u f(t, u)$ is continuous on $[0,1] \times[-M, M]$, there exists $C_{1}>0$ such that

$$
F(t, u) \leqslant \mu u f(t, u)+C_{1} \quad \forall t \in[0,1], u \in[-M, M]
$$

So, by $\left(\mathrm{H}_{3}\right)$, we obtain

$$
F(t, u) \leqslant \mu u f(t, u)+C_{1} \quad \forall t \in[0,1], u \in \mathbb{R}^{1}
$$

According to $\left(\mathrm{H}_{3}\right)$, for all $t \in[0,1]$ and $u \geqslant M$, we have

$$
\begin{aligned}
\left(\frac{F(t, u)}{u^{\nu}}\right)_{u}^{\prime} & =\frac{u^{v} f(t, u)-v u^{\nu-1} F(t, u)}{u^{2 v}} \\
& =\frac{u f(t, u)-v F(t, u)}{u^{v+1}} \geqslant 0 .
\end{aligned}
$$

Hence,

$$
\frac{F(t, u)}{u^{v}} \geqslant \frac{F(t, M)}{M^{v}} \geqslant M^{-v} \min _{t \in[0,1]} F(t, M)=C^{\prime}>0
$$

for all $t \in[0,1]$ and $u \geqslant M$. This implies that $F(t, u) \geqslant C^{\prime}|u|^{\nu}$ for all $t \in[0,1]$ and $u \geqslant M$. Similarly, we can prove that there is a constant $C^{\prime \prime}>0$ such that $F(x, u) \geqslant C^{\prime \prime}|u|^{\nu}$ for all $t \in[0,1]$ and $u \leqslant-M$. Since $F(t, u)-C_{2}|u|^{v}$ is continuous on $[0,1] \times[-M, M]$, there exists $C_{3}>0$ such that $F(t, u)-$ $C_{2}|u|^{\nu}>-C_{3}$ on $[0,1] \times[-M, M]$. Thus, we have

$$
F(t, u) \geqslant C_{2}|u|^{\nu}-C_{3} \quad \forall(t, u) \in[0,1] \times \mathbb{R}^{1},
$$

where $C_{2}=\min \left\{C^{\prime}, C^{\prime \prime}\right\}$.

Proof of Theorem 1. From $\left(\mathrm{H}_{1}\right)$, we have $A: P \rightarrow P$.

By $\left(\mathrm{H}_{2}^{\prime}\right)$, there exists a sufficiently small number $r>0$ such that

$$
\begin{aligned}
& f(t, u) \leqslant \pi^{4} u, \quad \forall(t, u) \in[0,1] \times[0, r] \\
& f(t, u)<\pi^{4} u, \quad \forall(t, u) \in[0,1] \times(0, r]
\end{aligned}
$$

Define $D=\{u \in P:\|u\|<r\}$ as an open convex subset of $P$ then

$$
\partial_{P} D=\{u \in P:\|u\|=r\} .
$$


For given $u \in H$, it follows from (i) of Remark 22 that

$$
\begin{aligned}
|K u(t)| & =\left|\int_{0}^{1} G(t, s) u(s) \mathrm{d} s\right| \\
& \leqslant \frac{1}{4} \int_{0}^{1}|u(s)| \mathrm{d} s \\
& \leqslant \frac{1}{4}\left(\int_{0}^{1}|u(s)|^{2} \mathrm{~d} s\right)^{1 / 2} \\
& =\frac{1}{4}\|u\|, \quad t \in[0,1] .
\end{aligned}
$$

This implies that

$$
\|K u\|_{C} \leqslant \frac{1}{4}\|u\|, \quad u \in H
$$

Thus,

$$
\|K u\|_{C} \leqslant \frac{1}{4}\|u\|=\frac{1}{4} r, \quad \forall u \in \partial_{P} D .
$$

Thereafter, for $u \in \partial_{P} D$, we have from (35) that

$$
\begin{aligned}
(A u)(t) & =\int_{0}^{1} G(t, s) f(t,(K u)(s)) \mathrm{d} s \\
& \leqslant \int_{0}^{1} G(t, s) \pi^{4}(K u)(s) \mathrm{d} s \\
& =\pi^{4}\left(K^{2} u\right)(t), \quad t \in[0,1] .
\end{aligned}
$$

Then, by (41), (36), and (iii) of Remark 22, we have

$$
\|A u\|<\pi^{4}\left\|K^{2} u\right\| \leqslant\|u\|, \quad \forall u \in \partial_{P} D
$$

namely, $A\left(\partial_{P} D\right) \subset D$.

Since $E \hookrightarrow H, \mathbf{f}: E \rightarrow E$ is a bounded continuous operator and $K$ is bounded linear operator, there exists $M^{\prime}>$ 0 such that

$$
\|\mathbf{f} K u\| \leqslant M^{\prime}\|u\|=M^{\prime} r, \quad \forall u \in \partial_{P} D .
$$

Thus, by (26), Hölder's inequality, and (43), we have

$$
\begin{aligned}
J(u) & =\frac{1}{2}\|u\|^{2}-\int_{0}^{1} F(t,(K u)(t)) \mathrm{d} t \\
& \geqslant \frac{1}{2} r^{2}-\mu \int_{0}^{1} f(t,(K u)(t))(K u)(t) \mathrm{d} t-C_{1} \\
& \geqslant \frac{1}{2} r^{2}-\mu\|\mathbf{f}(K u)\|\|K u\|-C_{1} \\
& \geqslant\left(\frac{1}{2}-\frac{\mu M^{\prime}}{\pi^{2}}\right) r^{2}-C_{1} \\
& =\text { constant, } \forall u \in \partial_{P} D .
\end{aligned}
$$

Choose $R>0$. Let $u_{R}(t)=R \sin \pi t, t \in[0,1]$. Obviously, $u_{R} \in P$. Since $v>2, L^{\nu}[0,1] \hookrightarrow H$, that is, there exists $C_{4}>0$ such that

$$
\|\cdot\| \leqslant C_{4}\|\cdot\|_{L^{\nu}[0,1]} .
$$

Consequently, we have from (27) that

$$
\begin{aligned}
J\left(u_{R}\right) & =\frac{1}{2}\left\|u_{R}\right\|^{2}-\int_{0}^{1} F\left(t,\left(K u_{R}\right)(t)\right) \mathrm{d} t \\
& \leqslant \frac{1}{4} R^{2}-\int_{0}^{1}\left[C_{2}\left|\left(K u_{R}\right)(t)\right|^{\nu}-C_{3}\right] \mathrm{d} t \\
& =\frac{1}{4} R^{2}-C_{2}\left\|K u_{R}\right\|_{L^{\nu}[0,1]}^{v}+C_{3} \\
& \leqslant \frac{1}{4} R^{2}-C_{2} C_{4}^{-v}\left\|K u_{R}\right\|^{v}+C_{3} \\
& =\frac{1}{4} R^{2}-C_{1} C_{4}^{-v} \pi^{-2 v}\left(\frac{1}{2}\right)^{v / 2} R^{v}+C_{2} .
\end{aligned}
$$

Since $v>2$, we have

$$
\lim _{R \rightarrow+\infty} J\left(u_{R}\right)=-\infty .
$$

Combining (47) and (44), we obtain that there exists $u_{1} \in$ $P \backslash D$ such that

$$
\inf _{u \in \partial_{P} D} J(u)>J(u)
$$

Now, all the conditions of Theorem 16 are satisfied. Therefore, Theorem 16 ensures that BVP (1) has at least a positive solution. The proof is completed.

Proof of Theorem 4. By the symmetry of $P$ and $-P$, it is easy to verify that all the conditions of Theorem 18 are satisfied. Theorem 18 ensures that BVP (1) has at least a positive solution and a negative solution. This completes the proof.

In order to prove Theorem 7 and Theorem 10, we need to construct convex subset $\mathscr{D}^{ \pm}$of $H$ and an operator $A$ satisfying the assumptions $\left(\mathrm{A}_{1}\right)$ and $\left(\mathrm{A}_{2}\right)$. We begin by transforming BVP (1) into the following equivalent boundary value problem:

$$
\begin{gathered}
u^{(4)}+m u=f_{1}(t, u(t)), \quad t \in[0,1] \\
u(0)=u(1)=u^{\prime \prime}(0)=u^{\prime \prime}(1)=0
\end{gathered}
$$

where $m>0$ and $f_{1}(t, u)=f(t, u)+m u$ for all $(t, u) \in[0,1] \times$ $\mathbb{R}^{1}$. Let $G_{1}(t, s)$ be Green's function for the linear boundary value problem

$$
-u^{\prime \prime}+m u=0, \quad u(0)=u(1)=0
$$

which is explicitly given by

$$
\begin{aligned}
& G_{1}(t, s) \\
& \quad= \begin{cases}(\omega \sinh \omega)^{-1} \cdot \sinh \omega s \cdot \sinh \omega(1-t), & 0 \leqslant s \leqslant t \leqslant 1, \\
(\omega \sinh \omega)^{-1} \cdot \sinh \omega t \cdot \sinh \omega(1-s), & 0 \leqslant t \leqslant s \leqslant 1,\end{cases}
\end{aligned}
$$


where $\omega=\sqrt{m}, \sinh x=\left(e^{x}-e^{-x}\right) / 2$ is the hyperbolic sine function. It is easy to verify that $G_{1}(t, s)>0$ for all $t, s \in[0,1]$. Define operators $K_{1}, \mathbf{f}_{1}: C[0,1] \rightarrow C[0,1]$, respectively, by

$$
\begin{gathered}
K_{1} u(t)=\int_{0}^{1} G_{1}(t, s) u(s) \mathrm{d} s, \quad t \in[0,1], \\
\forall u \in C[0,1], K_{1}^{2}=K_{1} \circ K_{1}, \\
\mathbf{f}_{1} u(t)=f_{1}(t, u(t)), \quad t \in[0,1], \quad \forall u \in C[0,1] .
\end{gathered}
$$

Obviously, $K_{1}$ and $\mathbf{f}_{1}$ have the same properties of $K$ and $\mathbf{f}$ as in Remark 22, respectively. Besides, $\left\|K_{1}\right\|=1 /\left(\pi^{2}+m\right)$ and $\left\|K_{1}^{2}\right\|=1 /\left(\pi^{4}+m\right)$.

Define a functional $J_{1}: H \rightarrow \mathbb{R}^{1}$ by

$$
J_{1}(u)=\frac{1}{2}\|u\|^{2}-\int_{0}^{1} F_{1}\left(t, K_{1} u(t)\right) \mathrm{d} t, \quad u \in H,
$$

where $F_{1}(t, u)=\int_{0}^{u} f_{1}(t, v) \mathrm{d} v$. Then, it is easy to see that $J_{1} \in$ $C^{1}\left(H, \mathbb{R}^{1}\right)$ with derivatives given by $J_{1}^{\prime}(u)=u-K_{1} \mathbf{f}_{1} K_{1} u$ for all $u \in H$. Set

$$
A_{1}=K_{1} \mathbf{f}_{1} K_{1} .
$$

Then, according to [7, Lemma 3.1], BVP (49) (i.e., BVP (1)) has a nontrivial solution in $C^{4}[0,1]$ if and only if the functional $J_{1}$ has a nontrivial critical point in $H$ (i.e., $A_{1}$ has a nontrivial fixed point in $H$ ). Similarly, since $K_{1}$ is a positive operator, if $v \in H$ is a positive/negative/sign-changing critical point of $J_{1}$, then $K_{1} v$ is a positive/negative/sign-changing solution of BVP (1) in $C^{4}[0,1]$, respectively.

Lemma 24. Assume that $\left(H_{1}\right)$ and $\left(H_{3}\right)$ hold. Then, the functional $J_{1}$ satisfies PS condition on $H$.

Proof. Suppose that $\left\{u_{n}\right\} \subset H$, and there exists $M_{1}>0$ such that $\left|J_{1}\left(u_{n}\right)\right| \leqslant M_{1}$ and

$$
J_{1}^{\prime}\left(u_{n}\right)=u_{n}-A_{1} u_{n} \longrightarrow 0 \quad \text { in } H \text { as } n \longrightarrow \infty
$$

Notice that

$$
\begin{aligned}
\left(J_{1}^{\prime}\left(u_{n}\right), u_{n}\right) & =\left(u_{n}-K_{1} \mathbf{f}_{1} K_{1} u_{n}, u_{n}\right) \\
& =\left\|u_{n}\right\|^{2}-\int_{0}^{1} f_{1}\left(t, K_{1} u_{n}(t)\right) K_{1} u_{n}(t) \mathrm{d} t
\end{aligned}
$$

and $\left\|K_{1}\right\|^{2}=1 /\left(\pi^{2}+m\right)^{2}$. It follows from (28) and the definition of $J_{1}$ that

$$
\begin{aligned}
M_{1} \geqslant & J\left(u_{n}\right)=\frac{1}{2}\left\|u_{n}\right\|^{2}-\int_{0}^{1} F_{1}\left(t, K_{1} u_{n}(t)\right) \mathrm{d} t \\
= & \frac{1}{2}\left\|u_{n}\right\|^{2}-\int_{0}^{1}\left(F\left(t, K_{1} u_{n}(t)\right)+\frac{m}{2}\left(K_{1} u_{n}(t)\right)^{2}\right) \mathrm{d} t \\
\geqslant & \frac{1}{2}\left\|u_{n}\right\|^{2}-\mu \int_{0}^{1} f\left(t, K_{1} u_{n}(t)\right) K_{1} u_{n}(t) \mathrm{d} t \\
& -\frac{m}{2} \int_{0}^{1}\left(K_{1} u_{n}(t)\right)^{2} \mathrm{~d} t-C_{1} \\
= & \frac{1}{2}\left\|u_{n}\right\|^{2}-\mu \int_{0}^{1} f_{1}\left(t, K_{1} u_{n}(t)\right) K_{1} u_{n}(t) \mathrm{d} t \\
& -\left(\frac{1}{2}-\mu\right) m\left\|K_{1} u_{n}\right\|^{2}-C_{1} \\
\geqslant & \frac{1}{2}\left\|u_{n}\right\|^{2}-\mu \int_{0}^{1} f_{1}\left(t, K_{1} u_{n}(t)\right) K_{1} u_{n}(t) \mathrm{d} t \\
& -\left(\frac{1}{2}-\mu\right) m\left\|K_{1}\right\|^{2}\left\|u_{n}\right\|^{2}-C_{1} \\
= & \left(\frac{1}{2}-\mu\right)\left\|u_{n}\right\|^{2}+\mu\left(J_{1}^{\prime}\left(u_{n}\right), u_{n}\right) \\
& -\left(\frac{1}{2}-\mu\right) \frac{m}{\left(\pi^{2}+m\right)^{2}}\left\|u_{n}\right\|^{2}-C_{1} \\
\geqslant & \left(\frac{1}{2}-\mu\right)\left(1-\frac{m}{\left(\pi^{2}+m\right)^{2}}\right)\left\|u_{n}\right\|^{2} \\
& -\mu\left\|J_{1}^{\prime}\left(u_{n}\right)\right\|\left\|u_{n}\right\|-C_{1}, \quad n=1,2, \ldots
\end{aligned}
$$

Since $J_{1}^{\prime}\left(u_{n}\right) \rightarrow 0$ as $n \rightarrow \infty$, there exists $N_{0} \in \mathbb{N}$ such that

$$
\begin{aligned}
M_{1} \geqslant & \left(\frac{1}{2}-\mu\right)\left(1-\frac{m}{\left(\pi^{2}+m\right)^{2}}\right)\left\|u_{n}\right\|^{2} \\
& -\left\|u_{n}\right\|-C_{1}, \quad n>N_{0} .
\end{aligned}
$$

This implies that $\left\{u_{n}\right\} \subset H$ is bounded. Since $A_{1}: H \rightarrow H$ is completely continuous, we have from (55) that $\left\{u_{n}\right\}$ has a convergent subsequence in $H$. Thus, $J_{1}$ satisfies PS condition on $H$.

Lemma 25. Assume that $\left(H_{1}\right)-\left(H_{5}\right)$ hold. Then, there exist $m>0$ and $\varepsilon_{0}>0$ such that

$$
A_{1}\left(\mathscr{D}_{\varepsilon}^{ \pm}\right) \subset \operatorname{int}\left(\mathscr{D}_{\varepsilon}^{ \pm}\right) \quad \text { for } \varepsilon \in\left(0, \varepsilon_{0}\right] \text {, }
$$

where $A_{1}$ is as defined in (54) and

$$
\mathscr{D}_{\varepsilon}^{ \pm}=\{u \in H: \operatorname{dist}(u, \pm P) \leqslant \varepsilon\} .
$$

Proof. As a consequence of $\left(\mathrm{H}_{1}\right)-\left(\mathrm{H}_{3}\right)$ and $\left(\mathrm{H}_{5}\right)$, there exists $m>0$ such that

$$
u f(t, u)+m u^{2}>0 \quad \forall t \in[0,1], u \in \mathbb{R}^{1} \text { with } u \neq 0 .
$$


By $\left(\mathrm{H}_{2}\right)$ and $\left(\mathrm{H}_{5}\right)$, there exist $\delta>0$ and $c_{1}>0$ such that

$$
\begin{array}{r}
|f(t, u)+m u| \leqslant\left(\pi^{4}+m-\delta\right)|u|+c_{1}|u|^{q-1} \\
\forall(t, u) \in[0,1] \times \mathbb{R}^{1} .
\end{array}
$$

Next, we show that there exists $\varepsilon_{0}>0$ such that

$$
\begin{aligned}
& \operatorname{dist}\left(A_{1} u, P\right)<\operatorname{dist}(u, P) \quad \text { as } 0<\operatorname{dist}(u, P) \leqslant \varepsilon_{0}, \\
& \operatorname{dist}\left(A_{1} u,-P\right)<\operatorname{dist}(u,-P) \quad \text { as } 0<\operatorname{dist}(u,-P) \leqslant \varepsilon_{0} .
\end{aligned}
$$

For any $u \in H$, define its positive part and negative part as follows:

$$
u^{+}(t)=\max \{u(t), 0\}, \quad u^{-}(t)=\min \{u(t), 0\} .
$$

Obviously, $u=u^{+}+u^{-}$for all $u \in H$. From (61), we have

$$
\begin{array}{ll}
f_{1}(t, u)=f(t, u)+m u>0 & \forall u>0 \\
f_{1}(t, u)=f(t, u)+m u<0 & \forall u<0 .
\end{array}
$$

This implies that

$$
\left(f_{1}(t, u)\right)^{-}=(f(t, u)+m u)^{-}=f\left(t, u^{-}\right)+m u^{-}
$$

for all $u \in H$. Since $K_{1}$ is a positive operator, $\left(K_{1} u\right)^{-}=K_{1} u^{-}$. So, we have from (67) that

$$
\begin{aligned}
\left(A_{1} u\right)^{-} & =\left(K_{1} \mathbf{f}_{1} K_{1} u\right)^{-}=K_{1}\left(\mathbf{f}_{1} K_{1} u\right)^{-} \\
& =K_{1} \mathbf{f}_{1}\left(K_{1} u\right)^{-}=K_{1} \mathbf{f}_{1} K_{1} u^{-}
\end{aligned}
$$

for all $u \in H$. For any $u \in H$, it follows that

$$
\begin{aligned}
\left|K_{1} u^{-}(t)\right| & =\left|\int_{0}^{1} G_{1}(t, s) u^{-}(s) \mathrm{d} s\right| \\
& \leqslant \max _{(t, s) \in[0,1] \times[0,1]} G_{1}(t, s) \int_{0}^{1}\left|u^{-}(s)\right| \mathrm{d} s \\
& \leqslant \max _{(t, s) \in[0,1] \times[0,1]} G_{1}(t, s)\left(\int_{0}^{1}\left|u^{-}(s)\right|^{2} \mathrm{~d} s\right)^{1 / 2} \\
& =\max _{(t, s) \in[0,1] \times[0,1]} G_{1}(t, s)\left\|u^{-}\right\|, \quad t \in[0,1] .
\end{aligned}
$$

Thereafter, for $t \in[0,1]$, we have

$$
\begin{aligned}
K_{1}\left(\left|K_{1} u^{-}(t)\right|^{q-1}\right) & \leqslant K_{1}\left[\left(\max _{(t, s) \in[0,1] \times[0,1]} G_{1}(t, s)\left\|u^{-}\right\|\right)^{q-1}\right] \\
& =c_{2}\left\|u^{-}\right\|^{q-1}
\end{aligned}
$$

where $c_{2}=\left(\max _{(t, s) \in[0,1] \times[0,1]} \int_{0}^{1} G(t, s) \mathrm{d} s\right)^{q}>0$.
Consider $u \in H$ and set $v=A_{1} u$. Then, by (68), (62), and (70), we obtain

$$
\begin{aligned}
\left\|v^{-}\right\|^{2} & =\int_{0}^{1}\left|\left(K_{1} \mathbf{f}_{1} K_{1} u\right)^{-}\right|^{2} \mathrm{~d} t \\
& =\int_{0}^{1}\left|K_{1}\left(f\left(t, K_{1} u^{-}\right)+m K_{1} u^{-}\right)\right|^{2} \mathrm{~d} t \\
& \leqslant \int_{0}^{1}\left|K_{1}\left(\left(\pi^{4}+m-\delta\right)\left|K_{1} u^{-}\right|+c_{1}\left|K_{1} u^{-}\right|^{q-1}\right)\right|^{2} \mathrm{~d} t \\
& =\int_{0}^{1}\left[\left(\pi^{4}+m-\delta\right)\left|K_{1}^{2} u^{-}\right|+c_{1} K_{1}\left(\left|K_{1} u^{-}\right|^{q-1}\right)\right]^{2} \mathrm{~d} t \\
& \leqslant \int_{0}^{1}\left[\left(\pi^{4}+m-\delta\right)\left|K_{1}^{2} u^{-}\right|+c_{1} c_{2}\|u\|^{q-1}\right]^{2} \mathrm{~d} t \\
& =\left\|\left(\pi^{4}+m-\delta\right)\left|K_{1}^{2} u^{-}\right|+c_{1} c_{2}\right\| u\left\|^{q-1}\right\|^{2} \cdot
\end{aligned}
$$

Therefore, we obtain that

$$
\begin{aligned}
\left\|v^{-}\right\| & \leqslant\left\|\left(\pi^{4}+m-\delta\right)\left|K_{1}^{2} u^{-}\right|+c_{1} c_{2}\right\| u\left\|^{q-1}\right\| \\
& \leqslant\left(\pi^{4}+m-\delta\right)\left\|K_{1}^{2} u^{-}\right\|+c_{1} c_{2}\left\|u^{-}\right\|^{q-1} \\
& \leqslant\left(1-\delta\left(\pi^{4}+m\right)^{-1}\right)\left\|u^{-}\right\|+c_{1} c_{2}\left\|u^{-}\right\|^{q-1} .
\end{aligned}
$$

Since $v=v^{+}+v^{-}$and $v^{+} \in P$,

$$
\operatorname{dist}(v, P) \leqslant\left\|v-v^{+}\right\|=\left\|v^{-}\right\| \text {. }
$$

Thus, it follows from (72) that

$$
\operatorname{dist}(v, P) \leqslant\left(1-\delta\left(\pi^{4}+m\right)^{-1}\right)\left\|u^{-}\right\|+c_{1} c_{2}\left\|u^{-}\right\|^{q-1} .
$$

For any $w \in P$, we have

$$
\left\|u^{-}\right\| \leqslant\|u-w\|
$$

In fact, let $I_{1}=\{t \in[0,1]: u(t) \geqslant 0\}=\left\{t \in[0,1]: u^{-}(t)=0\right\}$, $I_{2}=[0,1] \backslash I_{1}$. Then, $u^{-}(t)=u(t)<0$ for all $t \in I_{2}$. So,

$$
u(t)-w(t) \leqslant u(t)=u^{-}(t)<0
$$

for all $t \in I_{2}$, and then

$$
|u(t)-w(t)| \geqslant\left|u^{-}(t)\right|
$$

for all $w \in P$ and $t \in I_{2}$. Thus

$$
\|u-w\|^{2} \geqslant \int_{I_{2}}|u-w|^{2} \mathrm{~d} t \geqslant \int_{I_{2}}\left|u^{-}\right|^{2} \mathrm{~d} t=\left\|u^{-}\right\|^{2} .
$$

Combining (74) and (75), we deduce that

$$
\operatorname{dist}(v, P) \leqslant\left(1-\delta\left(\pi^{4}+m\right)^{-1}\right)\|u-w\|+c_{1} c_{2}\|u-w\|^{q-1}
$$


for all $w \in P$. Consequently,

$$
\begin{aligned}
\operatorname{dist}(v, P) \leqslant & \left(1-\delta\left(\pi^{4}+m\right)^{-1}\right) \operatorname{dist}(u, P) \\
& +c_{1} c_{2}(\operatorname{dist}(u, P))^{q-1} \\
< & \operatorname{dist}(u, P)+c_{1} c_{2}(\operatorname{dist}(u, P))^{q-1} .
\end{aligned}
$$

Since $q>2$, there exists $\varepsilon_{0}>0$ such that

$$
\operatorname{dist}(v, P)<\operatorname{dist}(u, P) \quad \text { as } 0<\operatorname{dist}(u, P) \leqslant \varepsilon_{0} \text {. }
$$

Similarly, we can find $\varepsilon_{0}>0$ small enough such that (64) holds. Up to now, these constants $m>0$ and $\varepsilon_{0}>0$ are as required. The proof is completed.

$$
\operatorname{Cr}\left(J_{1}\right) \cap\left(\mathscr{D}_{\varepsilon}^{+} \cap \mathscr{D}_{\varepsilon}^{-}\right)=\{\theta\}
$$

where $\theta$ denotes the function $u(t) \equiv 0$ for $t \in[0,1]$. Then, by (59) and [19, Lemma 3.2],

$$
\inf _{u \in \mathscr{D}_{\varepsilon}^{+} \cap \mathscr{D}_{\varepsilon}^{-}} J_{1}(u)=J_{1}(\theta)=0
$$

Define a path $h_{R}:[0,1] \rightarrow H$ as

$$
h_{R}(s)=R \cos \pi s \sin \pi t+R \sin \pi s \sin 2 \pi t, \quad R>0 .
$$

Then, $h_{R}(0)=R \sin \pi t$ and $h_{R}(1)=-R \sin \pi t$. Since

$$
\operatorname{dist}( \pm R \sin \pi t, \mp P)=\|R \sin \pi t\|=\frac{R}{\sqrt{2}},
$$

it is easy to see that $h_{R}(0) \in\left(\operatorname{int}_{H} \mathscr{D}_{\varepsilon}^{+}\right) \backslash \mathscr{D}_{\varepsilon}^{-}$and $h_{R}(1) \epsilon$ $\left(\right.$ int $\left._{H} \mathscr{D}_{\varepsilon}^{-}\right) \backslash \mathscr{D}_{\varepsilon}^{+}$as $R$ is large enough.
It follows from (29), (45), and a direct computation that

$$
\begin{aligned}
J_{1}\left(h_{R}(s)\right)= & \frac{1}{2}\left\|h_{R}(s)\right\|^{2}-\int_{0}^{1} F_{1}\left(t, K_{1} h_{R}(s)\right) \mathrm{d} t \\
\leqslant & \frac{1}{4} R^{2} \\
& -\int_{0}^{1}\left(C_{2}\left|K_{1} h_{R}(s)\right|^{v}-C_{3}+\frac{1}{2} m\left(K_{1} h_{R}(s)\right)^{2}\right) \mathrm{d} t \\
= & \frac{1}{4} R^{2}-C_{2}\left\|K_{1} h_{R}(s)\right\|_{L^{v}[0,1]}^{v} \\
& -\frac{1}{2} m\left\|K_{1} h_{R}(s)\right\|^{2}+C_{3} \\
\leqslant & \frac{1}{4} R^{2}-C_{2} C_{4}^{-v}\left\|K_{1} h_{R}(s)\right\|^{\nu} \\
& -\frac{1}{2} m\left\|K_{1} h_{R}(s)\right\|^{2}+C_{3} \\
= & \frac{1}{4} R^{2}-C_{2} C_{4}^{-v}[g(s)]^{v} R^{v}-\frac{1}{2} m[g(s)]^{2} R^{2}+C_{3} \\
\leqslant & C_{5} R^{2}-C_{6} R^{v}+C_{3},
\end{aligned}
$$

where

$$
\begin{gathered}
g(s) \\
=\left(\frac{\left(m^{2}+2 \pi^{2} m+\pi^{4}\right) \sin ^{2} \pi s+\left(m^{2}+8 \pi^{2} m+16 \pi^{4}\right) \cos ^{2} \pi s}{2 m^{4}+20 \pi^{2} m^{3}+66 \pi^{4} m^{2}+80 \pi^{6} m+32 \pi^{8}}\right)^{1 / 2} \\
C_{5}=\frac{1}{4}-\frac{1}{2} m \min _{s \in[0,1]}(g(s))^{2} \\
C_{6}=C_{2} C_{4}^{-v} \min _{s \in[0,1]}(g(s))^{v}>0 .
\end{gathered}
$$

Since $v>2$, we have

$$
\lim _{R \rightarrow+\infty} \max _{s \in[0,1]} J_{1}\left(h_{R}(s)\right)=-\infty .
$$

Therefore,

$$
\max _{s \in[0,1]} J_{1}\left(h_{R}(s)\right)<0=\inf _{u \in \mathscr{D}_{\varepsilon}^{+} \cap \mathscr{D}_{\varepsilon}^{-}} J_{1}(u)
$$

as $R$ is large enough.

Now, all the conditions of Theorem 20 are satisfied, and Theorem 20 ensures that BVP (1) has at least four solutions. According to the construction of $\mathscr{D}_{\varepsilon}^{ \pm}$and the locations of these solutions, we can easily see that one is zero, one is positive, one is negative, and one is sign changing. This completes the proof.

Proof of Theorem 10. From Lemmas 25 and 24, $J_{1}$ satisfies PS condition on $H$, and it is easy to see that $A_{1}$ and $\mathscr{D}_{\varepsilon}^{ \pm}$satisfy $\left(\mathrm{A}_{1}\right)$ and $\left(\mathrm{A}_{2}\right)$. From $\left(\mathrm{H}_{6}\right), J_{1}$ is an even functional. Next, we show that $\left(\mathrm{A}_{4}\right)$ holds. 
Denote that $H_{n}=\operatorname{span}\left\{e_{1}, e_{2}, \ldots, e_{n}\right\}$, where $e_{k}=\sqrt{2}$ $\sin k \pi t, k=1,2, \ldots, n$. For $u \in H_{n}$, there exist $a_{1}, a_{2}, \ldots, a_{n} \in$ $\mathbb{R}^{1}$ such that

$$
u=a_{1} e_{1}+a_{2} e_{2}+\cdots+a_{n} e_{n}, \quad r=\|u\|=\left(\sum_{i=1}^{n} a_{i}^{2}\right)^{1 / 2} .
$$

So, we have from (29) that

$$
\begin{aligned}
J_{1}(u) & =\frac{1}{2}\|u\|^{2}-\int_{0}^{1} F_{1}\left(t, K_{1} u(t)\right) \mathrm{d} t \\
& \leqslant \frac{1}{2} r^{2}-\int_{0}^{1}\left(C_{2}\left|K_{1} u(t)\right|^{\nu}-C_{3}+\frac{1}{2} m\left(K_{1} u(t)\right)^{2}\right) \mathrm{d} t \\
& =\frac{1}{2} r^{2}-C_{2}\left\|K_{1} u\right\|_{L^{v}[0,1]}^{\nu}-\frac{1}{2} m\left\|K_{1} u\right\|^{2}+C_{3} \\
& \leqslant \frac{1}{2} r^{2}-C_{2} C_{4}^{-v}\left\|K_{1} u\right\|^{\nu}-\frac{1}{2} m\left\|K_{1} u\right\|^{2}+C_{3} \\
& =\frac{1}{2} r^{2}-C_{2} C_{4}^{-v}\left\|\sum_{i=1}^{n} a_{i} \lambda_{i} e_{i}\right\|^{\nu}-\frac{1}{2} m\left\|\sum_{i=1}^{n} a_{i} \lambda_{i} e_{i}\right\|^{2}+C_{3} \\
& =\frac{1}{2} r^{2}-C_{2} C_{4}^{-v}\left(\sum_{i=1}^{n} a_{i}^{2} \lambda_{i}^{2}\right)^{v / 2}-\frac{1}{2} m\left(\sum_{i=1}^{n} a_{i}^{2} \lambda_{i}^{2}\right)+C_{3} \\
& \leqslant \frac{1}{2} r^{2}-C_{2} C_{4}^{-v}\left(\lambda_{n}^{2} \sum_{i=1}^{n} a_{i}^{2}\right)^{v / 2}-\frac{1}{2} m\left(\lambda_{n}^{2} \sum_{i=1}^{n} a_{i}^{2}\right)+C_{3} \\
& =\frac{1}{2}\left(1-m \lambda_{n}^{2}\right) r^{2}-C_{2} C_{4}^{-v} \lambda_{n} r^{v}+C_{3},
\end{aligned}
$$

where $\lambda_{i}$ denotes the $i$ th eigenvalue of $K_{1}$. Consequently,

$$
\lim _{\|u\| \rightarrow+\infty} J(u)=-\infty, \quad u \in H_{n} .
$$

This implies that

$$
\sup _{u \in H_{n} \backslash B_{n}} J_{1}(u) \leqslant \alpha_{1}<\alpha_{0}=0=\inf _{u \in \mathscr{D}_{\varepsilon}^{+} \cap \mathscr{D}_{\varepsilon}^{-}} J_{1}(u),
$$

where $B_{n}=\left\{u \in H_{n}:\|u\|_{H} \leqslant R_{n}\right\}$. Up to now, all the conditions of Theorem 21 are satisfied. So, BVP (1) has infinitely many solutions in

$$
\mathscr{M}=\partial_{H} C_{H}(\mathcal{O}) \backslash\left(C_{H}\left(\operatorname{int}_{H} \mathscr{D}_{\varepsilon}^{+}\right) \cup C_{H}\left(\operatorname{int}_{H} \mathscr{D}_{\varepsilon}^{-}\right)\right) .
$$

Obviously, all of them are sign changing. This completes the proof.

\section{Acknowledgments}

The authors would like to thank the anonymous reviewers for their helpful comments. This paper was supported by National Natural Science Foundation of China (11101253 and 10826081), the Fundamental Research Funds for the Central Universities (Program no. GK200902046), and the Scientific Research Foundation of Xi'an University of Science and Technology (no. 200843).

\section{References}

[1] Z. Bai and H. Wang, "On positive solutions of some nonlinear fourth-order beam equations," Journal of Mathematical Analysis and Applications, vol. 270, no. 2, pp. 357-368, 2002.

[2] R. Y. Ma and H. P. Wu, "Positive solutions of a fourth-order twopoint boundary value problem," Acta Mathematica Scientia. Series A, vol. 22, no. 2, pp. 244-249, 2002.

[3] Y. Li, "Positive solutions of fourth-order periodic boundary value problems," Nonlinear Analysis. Theory, Methods \& Applications, vol. 54, no. 6, pp. 1069-1078, 2003.

[4] Y. Li, "Positive solutions of fourth-order boundary value problems with two parameters," Journal of Mathematical Analysis and Applications, vol. 281, no. 2, pp. 477-484, 2003.

[5] B. Liu, "Positive solutions of fourth-order two point boundary value problems," Applied Mathematics and Computation, vol. 148, no. 2, pp. 407-420, 2004.

[6] Q. Yao, "Positive solutions for eigenvalue problems of fourthorder elastic beam equations," Applied Mathematics Letters, vol. 17, no. 2, pp. 237-243, 2004.

[7] F. Li, Q. Zhang, and Z. Liang, "Existence and multiplicity of solutions of a kind of fourth-order boundary value problem," Nonlinear Analysis. Theory, Methods \& Applications, vol. 62, no. 5, pp. 803-816, 2005.

[8] G. Han and F. Li, "Multiple solutions of some fourth-order boundary value problems," Nonlinear Analysis. Theory, Methods \& Applications, vol. 66, no. 11, pp. 2591-2603, 2007.

[9] Y. Li, "Multiply sign-changing solutions for fourth-order nonlinear boundary value problems," Nonlinear Analysis. Theory, Methods \& Applications, vol. 67, no. 2, pp. 601-608, 2007.

[10] C. Bai, "Triple positive solutions of three-point boundary value problems for fourth-order differential equations," Computers \& Mathematics with Applications, vol. 56, no. 5, pp. 1364-1371, 2008.

[11] G. Han and Z. Xu, "Multiple solutions of some nonlinear fourthorder beam equations," Nonlinear Analysis. Theory, Methods \& Applications, vol. 68, no. 12, pp. 3646-3656, 2008.

[12] F. Li, Y. Zhang, and Y. Li, "Sign-changing solutions on a kind of fourth-order Neumann boundary value problem," Journal of Mathematical Analysis and Applications, vol. 344, no. 1, pp. 417428, 2008.

[13] Y. Yang and J. Zhang, "Infinitely many mountain pass solutions on a kind of fourth-order Neumann boundary value problem," Applied Mathematics and Computation, vol. 213, no. 1, pp. 262271, 2009.

[14] Y. Yang and J. Zhang, "Nontrivial solutions for some fourth order boundary value problems with parameters," Nonlinear Analysis. Theory, Methods \& Applications, vol. 70, no. 11, pp. 3966-3977, 2009.

[15] Y. Yang and J. Zhang, "Existence of infinitely many mountain pass solutions for some fourth-order boundary value problems with a parameter," Nonlinear Analysis. Theory, Methods \& Applications, vol. 71, no. 12, pp. 6135-6143, 2009.

[16] D. Guo, Nonlinear Functional Analysis, Shandong Science and Technology Press, Ji'nan, China, 2nd edition, 2001.

[17] D. J. Guo and V. Lakshmikantham, Nonlinear Problems in Abstract Cones, vol. 5, Academic Press, New York, NY, USA, 1988.

[18] M. Conti, L. Merizzi, and S. Terracini, "Remarks on variational methods and lower-upper solutions," Nonlinear Differential Equations and Applications, vol. 6, no. 4, pp. 371-393, 1999. 
[19] T. Bartsch, Z. Liu, and T. Weth, "Nodal solutions of a $p$ Laplacian equation," Proceedings of the London Mathematical Society, vol. 91, no. 1, pp. 129-152, 2005.

[20] P. H. Rabinowitz, Minimax Methods in Critical Point Theory with Applications to Differential Equations, vol. 65 of CBMS Regional Conference Series in Mathematics, American Mathematical Society, Providence, RI, USA, 1986.

[21] Z. Liu and J. Sun, "Invariant sets of descending flow in critical point theory with applications to nonlinear differential equations," Journal of Differential Equations, vol. 172, no. 2, pp. 257-299, 2001.

[22] J. Sun and X. Xu, "Decreasing flow invariant sets and fixed points of quasimonotone increasing operators," Proceedings of the American Mathematical Society, vol. 129, no. 9, pp. 26632669, 2001. 


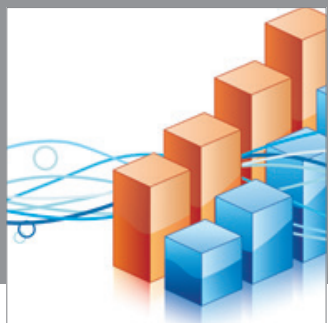

Advances in

Operations Research

mansans

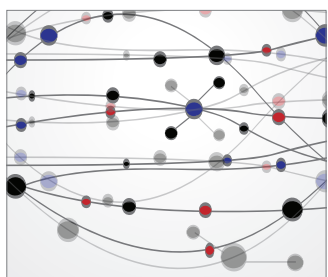

The Scientific World Journal
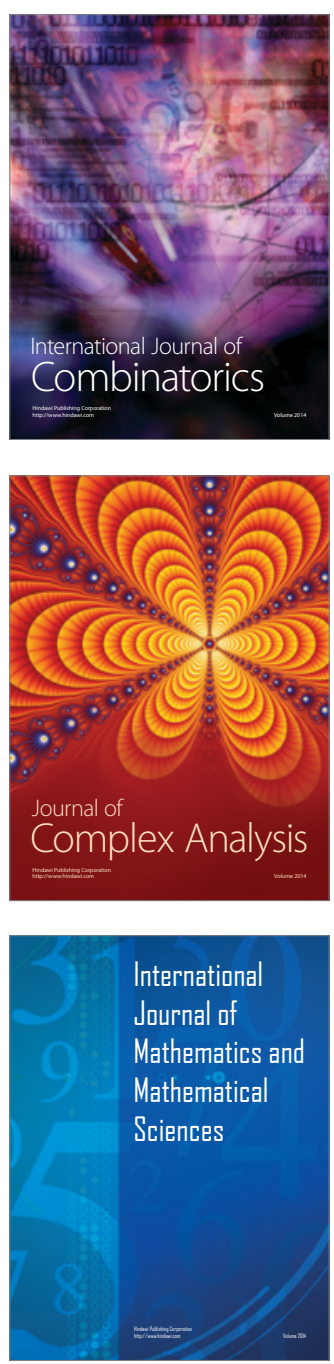
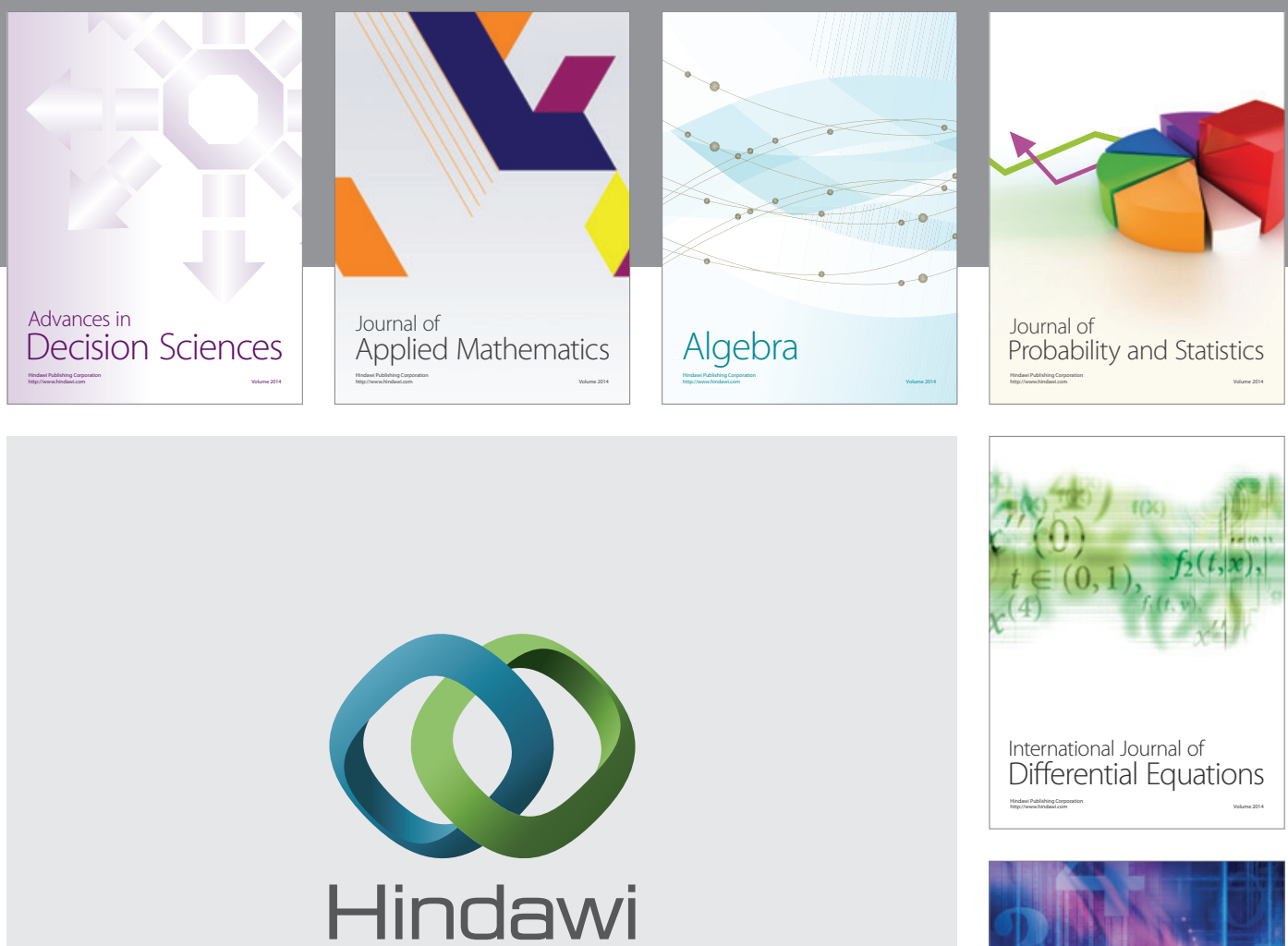

Submit your manuscripts at http://www.hindawi.com
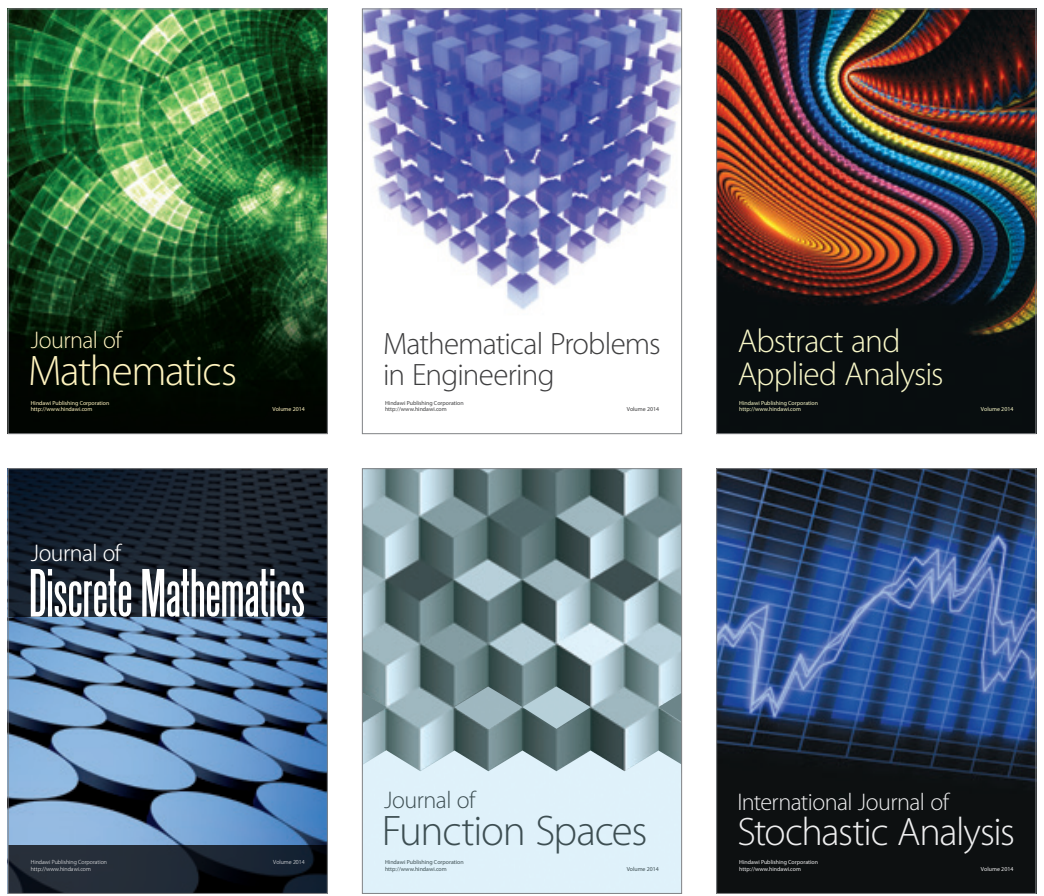

Journal of

Function Spaces

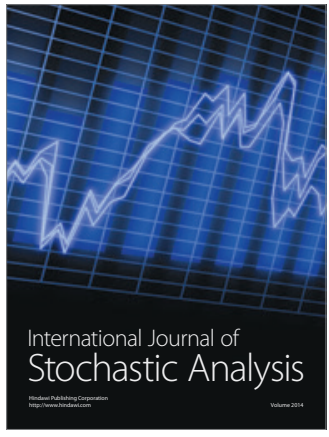

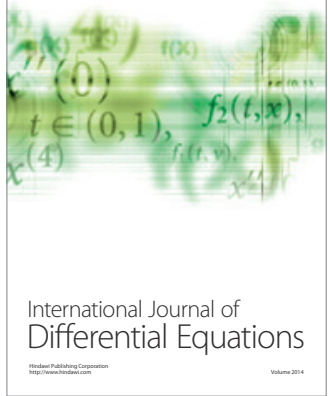
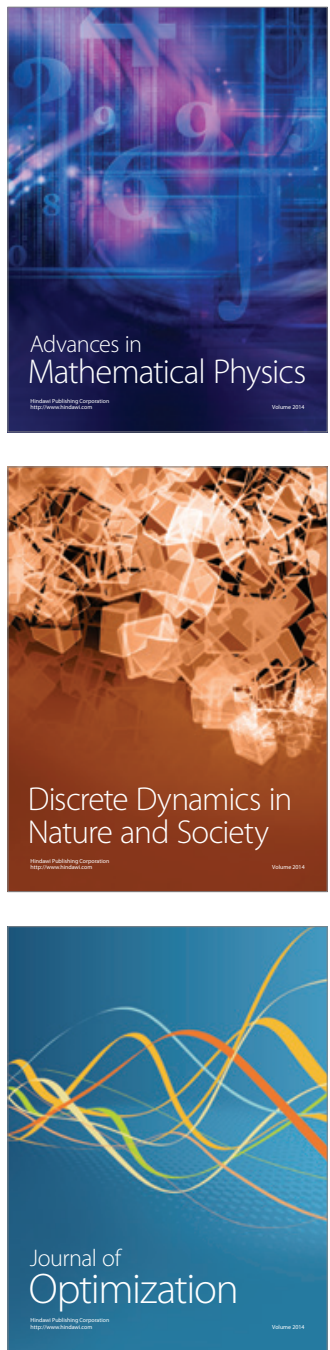